\title{
SEGUNDA MESA REDONDA
}

Doctor Aparicio: Iniciamos la mesa redonda con la siguiente pregunta:

¿Existe algún estudio estadístico en Latinoamérica para determinar los límites normales de la aparición de la Menarquia y cuáles son esas edades?

Doctor Di Paola: Bueno; en la Argentina se ha hecho un estudio estadístico con respecto a este problema. En la ciudad de Buenos Aires se llevó a cabo, hace ya muchos años, un estudio entre todas las escolares, que hemos citado ya en nuestro libro de Endocrinología, y la edad media de la aparición de la primera menstruación en la ciudad dicha, es a los trece años y medio.

Doctor Guerrero: El mismo estudio se hizo en México, y el doctor González Gutiérrez, aquí presente, llegó a las mismas conclusiones. La edad de la menarca es más o menos a los trece o catorce años. Pero en nuestro país hay una notable diferencia entre las mujeres que viven cerca del trópico en que aparece la menarca entre los once y los doce años, mientras que en las que viven en el altiplano aparece más tardíamente. El estudio de Gutiérrez Gonzá- lez era de la población de Guadalajara, que está a unos mil doscientos metros sobre el nivel del mar.

Doctor López-Escobar: La doctora Cesarina de Borrero hace la anotación de que en su tesis de grado, hecha en 1953, ella hizo una estadística en Colombia sobre la edad de la Menarquia, que fue la de trece años y medio.

Pregunta: Doctor Guerrero, según su programa diagnóstico terapéutico, ¿qué valor le da usted a la prueba del embarazo con progesterona estrógenos?

Doctor Guerrero: Yo dije que la asociación de progesterona estrógenos en la técnica llamada de Zondek, es decir, dos ampolletas que tienen $x$ cantidad de progesterona y de estrógenos, lo que actúa es la progesterona. Porque nada más por sentido común, los estrógenos que están allí no son capaces de levantar un endometrio. No sirven de nada. Sirve la progesterona sola; e insisto en que los progestágenos no, porque los progestágenos no sirven para prueba diagnóstica.

Doctor Di Paola: Solamente para manifestar mi absoluta concordancia 
con lo que dice el doctor Guerrero. Nosotros nunca comprendimos por qué la industria Farmacéutica se obstinaba en preparar este producto. Claro está que en todos estos casos, cuando se utiliza la prueba es cuando la mujer cree que está embarazada. Entonces siempre es una amenorrea leve, con estrógenos, porque el retraso menstrual es de pocos días. En todos estos casos es ya una amenorrea con estrógenos; a nadie se le va a ocurrir hacer esta prueba diagnóstica para el caso de la amenorrea grave, es decir, de la amenorrea sin estrógenos, porque en este caso se trata ya de una amenorrea de larga duración. Entonces naturalmente hay que recurrir a la prueba de estrógenos. Pero a pesar de esto, y de que se nos consultó oportunamente por una de las casas más importantes que ha hecho este producto, y de nuestra opinión en contra, se obstinaron en hacer este producto, $\mathrm{y}$ han tenido suerte porque al parecer desde el punto de vista comercial creo que han tenido mucho éxito.

Pregunta: Doctor Guerrero, ¿cómo explica usted el mecanismo fisiopatológico de las amenorreas por sinequias uterinas pequeñas? Resultados médicos al respecto.

Doctor Guerrero: No podiía yo diferenciar entre pequeñas y grandes. Si por grandes se entiende la desaparición total de la cavidad uterina, entonces se actúa como si fuera una histerectomía, habiendo útero: Algunas que otras veces lo hemos visto. Yo mismo lo he producido y lo tengo publicado, en unas miomectomías con útero muy grande con una cavidad endometrial larga, que me empeñé en conservar el endometrio que después se plegó, y al plegarse quedó un pedacito en la parte inferior. Pero con esta expresión de sinequias pequeñas quizá se quieran referir a que encuentran pequeñas zonas de endometrio, y entonces el que pregunta esto me dirá: Si hay endometrio, ¿por qué no hay sangre? Entonces esto me lo explico en esta forma. A veces esas sinequias cierran la cavidad uterina. La zona de endometrio funcionante, aunque esté presente es tan escasa, que no alcanza a crear regla. Son mujeres que pueden menstruar gotas algún tiempo y actuar a la manera de una endometriosis y entonces producir no sólo dismenorrea, sino pequeñas zonas de retención que acaban por fundir y por pegar el útero, y las que eran pequeñas sinequias se convierten en una sinequia total.

Doctor Di Paola: Esta vez no estoy de acuerdo con mi querido amigo el doctor Guerrero. Este problema de las amenorreas uterinas por sinequia, tiene una importancia extraordinaria, porque en realidad desde hace algún tiempo, después de los estudios de Aschermann, se ha conseguido un nuevo tratamiento muy fácil y muy efectivo de este tipo de amenorreas, efectuando la dilatación del cuello uterino. La dilatación y un poco el desbridamiento de la cavidad. Aschermann está perfectamente convencido de que el efecto se logra simplemente con la dilatación del cuello. Nadie sabe por qué se curan estas amenorreas. Porque lo interesante es que cuando nosotros hemos hecho muchas veces biopsias de endometrios simultáneas, hemos encontrado endometrio pro- 
gestacional, pero la mujer estaba en amenorrea. Otras veces hemos encontrado endometrios estrogénicos. Pero basta que se haga la dilatación del cuello y a veces una simple histerometría, para que aparezca la menstruación a los pocos días. Y a veces con hacer una simple histerografía, con lo cual se comprueba el diagnóstico, que siempre se debe hacer en estos casos, sobre todo cuando las amenorreas aparecen después de un raspado, basta para que aparezca la menstruación.

En nuestro ambiente, estos son hechos muy conocidos y muy utilizados. Ultimamente el doctor Bruno ha hecho un trabajo muy interesante en donde ha reunido una gran cantidad de casos, esto es un tratamiento que lo practicamos habitualmente y lo recomendamos calurosamente. No es un tratamiento quirúrgico; es, digámoslo así, una maniobra de orden médico que se puede hacer en el consultorio y que puede producir un pequeño dolor, pero que no es demasiado fuerte, como es una simple dilatación. La explicación de esto, como lo he dicho y lo repito, no se sabe; pero lo evidente es que el resultado se consigue con toda facilidad, procediendo así.

Doctor Guerrero: No es que yo esté en desacuerdo con el doctor Di Paola. Lo que pasa es que yo contesté a la pregunta de cómo me podía explicar la amenorrea en las pequeñas sinequias; no cómo podía tratarlas. Entonces, estoy de acuerdo en lo que se refiere a tratamiento, muchas histerografías curan esto. Pero sí quiero hacer una aclaración y es que empezó el aspecto de sinequias abarcando solamente a sinequias post-legrado. Después se habló de sinequias post-partum en procesos inflamatorios e infecciosos. Y desde luego que también estamos de acuerdo con que hay sinequias por inflamación y destrucción del endemetrio, por tuberculosis endometrial. Entonces, se recuperan aquellas que son debidas a destrucción o a adherencias inflamatorias, pero en donde quedan tejidos endometriales activos susceptibles de volver a funcionar. Pero aquellas en donde hay una destrucción total del endometrio por una infección tuberculosa, me parece que esas son irrecuperables a pesar de que se hagan todos los tratamientos.

Doctor Murray: Hay un hecho importante en las sinequias uterinas y es el estado de la función ovárica. Hemos tenido ocasión de hacer estudios de la función ovárica en varias enfermas con sinequia, y recuerdo en tres de ellas particularmente, en que había ciclos monofásicos, endometrios persistentemente monofásicos. Creemos que no es entonces una patogenia de orden local, simplemente en el útero, sino que interviene en ello el ovario, o posiblemente la hipófisis.

El mecanismo, como ha dicho bien el doctor Di Paola, es desconocido, y como hipótesis podría plantearse la posibilidad de un reflejo, vía hipofisaria o directamente hacia el ovario.

Pregunta: Profesor Di Paola, ¿qué importancia tienen las gonadotrofinas en el tratamiento de la amenorrea y en su diagnóstico? 
Doctor Di Paola: Bueno, nosotros nos hemos ocupado desde hace mucho tiempo de establecer con cierta exactitud el lugar que les corresponde a las gonadotrofinas tanto en el diagnóstico, como en el tratamiento de las amenorreas. Reuniendo así, en muchos años, alrededor de 100 casos, que hemos presentado el año pasado al Congreso Mundial de Viena y llegamos a la conclusión de que, desde el punto de vista del diagnóstico de hacer las pruebas de gonadotrofinas, que no es como el tratamiento que propone Hablen, sino como el que propone Riedberg, es decir dosis muy grandes; hacer 15.000 unidades de gonadotrofina sérica más 5 o 6.000 unidades de gonadotrofina ordinaria en un lapso de nueve días, entonces la respuesta se obtiene entre ocho y doce días después de la última inyección. $\mathrm{Si}$ es positiva, quiere decir que la falla estaba en la hipófisis; si es negativa, quiere decir que la falla estaba en el ovario.

Esta prueba nos la hemos puesto hace muchos años y la hemos hecho por lo menos unas 100 veces. Algunas veces hemos tenido el inconveniente de producir lo que hemos llamado Respuesta Inusitada, de los Ovarios, vale decir, que en caso de insuficiencia hipofisiaria, la respuesta de esta dosis tan grande de gonadotrofina ha sido realmente seria porque se ha producido un aumento de tamaño entre 8 a 10 o 15 veces su tamaño normal, pero apareciendo como dos grandes tumores en la cavidad abdominal, con dolores intensos y con temperatura. El profesor Figueroa, de Rosario, tiene un caso en el cual la enferma murió, porque se interpretó como un tumor ovárico, se hizo una laparo- tomía; la mujer entró en anuria; hizo una nefrosis en el nefrón distal, y la enferma murió. Nosotros tenemos 5 casos; en los cuales no tuvimos mayores inconvenientes; esperamos que pasara la reacción; no fue necesario operarla, en ellas se interpretó bien el caso y todo pasó bien sin inconveniente. Pero nosotros creemos más conveniente la dosificación de gonadotrofinas, para establecer la participación de la hipófisis en el mecanismo de la amenorrea.

La dosificación de gonadotrofinas urinarias es para nosotros uno de los elementos de diagnóstico más importantes, si no el más importante en el estudio de la amenorrea. Y creemos que es indispensable hacerlo en todos los casos. Desgraciadamente no en todos los ambientes se puede hacer con facilidad. Porque no solamente no se indica en el caso de estar aumentadas las gonadotrofinas, que la amenorrea es de causa ovárica, o en el caso de estar disminuídas, que es de causa hipofisiaria, es decir, que desde el punto de origen de la patogenia de la amenorrea, ya que no podemos ir de la etiología, sino que además, y esto es una aplicación práctica que para mí tiene una importancia muy grande, nos ayuda a hacer el pronóstico. Mientras las gonadotrofinas no estén aumentadas, siempre podemos guardar alguna esperanza de que la amenorrea se va a resolver. Porque muchos de estos casos son amenorreas hipotalámicas, sicógenas, y que se mantienen en esa situación por circunstancias que no alcanzamos a comprender bien con nuestra sicoterapia, sencilla y simple; que tal vez haciendo una sicoterapia profunda altamente especia- 
lizada o talvez el sicoanálisis, se podrían esos casos resolver mejor, pero como son esas cosas tan complicadas y que tardan tanto tiempo y que son tan numerosas, muchas veces no se puede aconsejar a una niña de 20 años, que se sicoanalice por una causa así. De modo que si tenemos la seguridad de que el dosaje de las gonadotrofinas es normal, podemos siempre tener la esperanza de que esa amenorrea se va a resolver y mantener el estado trófico del aparato genital hasta que en un momento dado otro factor emocional, ya sea evidente o solapado, se restablezca normalmente el ritmo menstrual, de modo que esa es la importancia que le atribuímos al dosaje de las gonadotrofinas, en el estudio, el diagnóstico, el pronóstico de la amenorrea.

Pregunta: Doctor López, ¿cómo actúa el parasitismo para producir amenorreas?

Doctor López-Escobar: Yo creo que el parasitismo en sí solo no actúa; actúa el parasitismo intenso de aquellas personas que llegan a una desnutrición y a una anemia intensa por un alto parasitismo; y yo me he empeñado en insistir tanto dentro de la cátedra como entre nosotros, porque aquellos países que no tienen parásitos se olvidan de este tipo de problemas, como se olvidan de que existen desnutriciones tan intensas que pueden llegar a producir amenorreas.

Pregunta: Doctor Guerrero, en su opinión, ¿cuál sería el mecanismo de la amenorrea en las portadoras de fístulas urogenitales?
Doctor Guerrero: Yo no he visto ningún caso de amenorrea por fístula urogenital; quisiera saber si alguien en el auditorio tiene experiencia de esto.

Doctor López-Escobar: El doctor Bussamara va a presentar su trabajo al respecto mañana, luego sería inútil pedirle que lo hiciera hoy; sin embargo, en el servicio nosotros hemos tenido inmensa cantidad de fístulas grandes con amenorrea que se han prácticamente producido por un grado de desnutrición extremo.

Doctor Bedoya: Nosotros tenemos puntos muy interesantes, entre ellos un caso de amenorrea por fístula vésico-vaginal, que es el siguiente:

La enferma consultó por la amenorrea, no por la fístula. Desde más o menos tres años de un parto ella era portadora de su fístula, y así desde esa fecha, más o menos tres meses después apareció la amenorrea, que según lo dijo la mesa directiva se puede catalogar dentro de las de tipo nutricional.

Doctor Guerrero: Yo creo que entonces esto entra dentro del capítulo de amenorreas por enfermedades graves serias; la enferma no come bien porque tiene miedo de que orina, se deshidrata, respectivamente viene hipoproteinemia. Creo que en todos estos casos, ojalá que en la ponencia de mañana nos digan si estas enfermas tienen un ciclo artificial menstrual o no para saber si el endometrio es receptor o no y que entonces es de causa hipofisiaria u ovárica.

Doctor Di Paola: Yo me voy a permitir nombrar un caso que hemos te- 
nido y que es todo lo contrario. Es lo que se llama una Menouria; sí, una mujer con una fístula vésico-uterina, que menstruaba a través de la uretra. Como es tan raro, y como en el Congreso se va a tratar el tema de fístula, es interesante consignarlo. Durante 20 años menstruó a través de la uretra, luego después de una cesárea se le hizo una histerectomía y curó la fístula perfectamente.

Pregunta: Doctor Murray, ¿usó dosis total de la roengenterapia estimuladora sobre hipófisis y ovario en las amenorreas? Epoca de la respuesta.

Doctor Murray: En nuestro país ya hace unos cuantos años se encaró con gran entusiasmo la roengenterapia ovárica. Este entusiasmo ha decaído mucho en favor de la resección parcial de ovarios. Hemos tenido resultados muy buenos con radioterapia a pequeñas dosis y creemos que la indicación en estos casos debe basarse en una existencia de una estrogenoterapia normal.

Hemos visto dos casos en los cuales hubo consecuencias desagradables: la enferma entró en amenorrea a consecuencia de una irradiación ovárica. Esos dos casos nos han limitado nuestros entusiasmos, de modo que actualmente preferimos hacer un estudio preliminar, en primer lugar de los diez y siete cetosteroides para localizar la función alterada, si es en el ovario o es en el suprarrenal; en segundo caso recurrimos a la resección parcial, pero la irradiación tratamos de disminuírla al minimum posible; hay ya demasiados motivos de irradiación, para agregarle otra a la enferma.
Doctor Guerrero: Tengo aquí dos argentinos: en el Congreso de Sao Paulo se presentó esta pregunta, nos pronunciamos en contra. Sobre todo cuando. un grupo de argentinos presentó su experiencia en este sentido. Se había presentado un trabajo de los entonces médicos en Cátedra, entusiastas, y otro trabajo, de los médicos argentinos despojados de sus Cátedras en aquel entonces, quienes hicieron una estadística de enfermas puestas debajo de aparatos Rayos X sin pasar rayos, siendo los resultados los mismos. Está en actas del Congreso de Sao Paulo.

Doctor Di Paola: Tengo que hablar en primer lugar porque el doctor Guerrero se acuerda que en el Congreso de Sao Paulo se dijo esto, pero se olvida que en el último Congreso se habló de este tema y yo pude disertar sobre este asunto y me ocupé especialmente al respecto. Como dice el doctor Murray, en la Argentina se ha usado este procedimiento; es y será usado ese procedimiento. Yo creo sinceramente que es el mejor tratamiento: para la amenorrea leve, es decir, para la amenorrea con estrógenos. No hay ningún tratamiento mejor que este, pero lo usamos cada vez menos, por la misma razón que en México. Estamos sicológicamente influídos por las ponencias y los comunicados de los consejos de la Energía Atómica de todos los países más adelantados del mundo, U. R. S. S., U. S. A., e Inglaterra, donde se pone en guardia contra los peligros de las: radiaciones. Yo estuve una vez en los U. S. A., en que se tenía miedo de ver T. V. porque decían que de ella se desprendían radiaciones. Vuelvo a insistir 
y lo sostengo y está de acuerdo conmigo el doctor Rock, de Boston, que como ustedes saben, es una autoridad en la materia, pero quien tampoco usa las radiaciones; él estaba de acuerdo conmigo en que era el mejor tratamiento pero que él lo usaba cada vez menos. Efectivamente nosotros tenemos una estadística bastante importante, con el $80 \%$ de casos curados y con embarazos. Pero en los últimos años mandaré hacer anualmente entre todas las enfermas de este tipo que yo veo, a dos o tres nada más, casos en los cuales no hay otra solución; pero recuerdo, como lo dijo el doctor Murray, que es obligación que la enferma tenga una amenorrea leve, como nosotros la llamamos, y no decimos primer grado, para no confundir con la primaria. Insistimos en esto hace como veinte años, pero nadie nos hace caso. Por eso la llamamos grave cuando no tiene estrógenos y leve cuando sí hay estrógenos; $\mathrm{y}$ en estos casos los resultados son realmente extraordinariamente buenos.

Doctor Guerrero: Perdonen, pero es que creo que es una cuestión suficientemente importante, para el auditorio, que nos pongamos de acuerdo a escuchar nuestros puntos de vista e insistir en que aún esas pocas indicaciones que existen requieren un radioterapeuta, muy competente, porque de otra manera hacemos muchas amenorreas definitivas, es decir, super-graves.

Pregunta: Doctor Murray, en qué caso de amenorrea está indicado el tratamiento meramente quirúrgico; creo que esta pregunta se hace con exclusión de tumores.
Doctor Murray: Yo ya lo he dicho que se trata de los casos de ovarios poliquísticos con ciclo monofásico. Es conveniente discutir, y es que si en esos casos se requiere que haya un estrogenismo normal o si se puede hacer la resección parcial de ovarios en el caso en que haya un hipoestrogenismo. Indudablemente y yo lo he comprobado, en los casos en que hay hiperestrogenismo o un estrogenismo anormal los resultados son magníficos. En cambio he tenido fallas cuando hay un hipoestrogenismo revelado por una hipotrofia vaginal que ha dado como consecuencia una amenorrea definitiva, en la enferma. En esos casos se ha obtenido sin embargo una pequeña metrorragia posterior a consecuencia de intervenciones, pero nuevamente amenorrea. Yo soy partidario de que haya un nivel suficiente de estrógenos igualmente con la radioterapia. Por otra parte, si hay una amenorrea que coincide con la hipoplasia, mi concepto es tratar en primer lugar la hipoplasia y luego la amenorrea, es decir, dejar en segundo lugar el factor ovárico.

Pregunta: Doctor Guerrero, ¿qué utilidad práctica tiene la citología vaginal en el estudio de las amenorreas?

Doctor Guerrero: La pregunta dice práctica, desde luego que no le resto su valor, pero yo la uso en los casos ya mencionados, el $10 \%$ de los casos de amenorrea, cuando estoy inseguro del valor real que tiene el ovario, cuando los factores terapéuticos que mostré a ustedes no son suficientes, para hacerlos responder, para medir la capacidad ovárica de fondo. Se ha mencionado 
mucho que si la vaginal o la urológica; creo que es indistinto una de otra si el que la lee lo sabe leer.

Doctor Avilés: Nosotros somos muy antiguos en el empleo del estudio de la citología vaginal porque la usamos desde 1932 o 33 . Hemos tenido bastante experiencia en esto. Estamos de acuerdo con el doctor Guerrero porque nos parece que es un tratamiento muy trabajoso, que hay que hacerlo diariamente durante un tiempo prolongado para que dé resultado. Entonces este procedimiento, por lo complicado y por todos los trabajos que ocasiona, no justifica su aplicación. Sin embargo, es innegable su importancia para establecer si la amenorrea es leve o grave, porque la cristalización del moco cervical que nosotros hemos propugnado para hacer el diagnóstico diferencial es evidentemente un procedimiento mucho más fácil, pero no siempre hay unas enfermas con amenorrea y cantidades suficientes de moco como para poder asegurar el diagnóstico de cristalización o no. La biopsia del endemetrio es un buen procedimiento, pero se produce dolor y como muy a menudo las mujeres que tienen amenorrea son niñas adolescentes, en las cuales no se puede hacer biopsia de endometrio, en esos casos la citología vaginal o urinaria, sobre todo la urinaria en las niñas vírgenes es insustituíble. Sólo con la prueba de la progesterona se puede sustituír. Nosotros la utilizamos y hemos simplificado su empleo. Durante el mes que dura el estudio de la amenorrea la hacemos 4 veces; una vez por semana. ¿Y por qué hacemos esto? Porque creemos que aun dentro de las amenorreas, sí se puede diagnosticar con los 4 cua- dros citológicos de las diferentes tomas, a razón de una por semana, que se han hecho durante el mes; que si hay cierta oscilación en la respuesta uretro-vesical, el pronóstico es mejor que si no la hay. Se prefiere en las muchachas jóvenes hacer el urocitograma y no el colpocitograma por razones ya expresadas. La citología se ha difundido porque es mucho más fácil que hacer dosificaciones hormonales, y en nuestros países es más fácil instalar un laboratorio de citodiagnóstico que no uno de dosificaciones hormonales.

\section{EXPERIENCIAS DE LA MESA SOBRE EL INJERTO OVARICO EN LAS AMENORREAS DE CAUSA OVARICA}

Doctor Di Paola: Hace unos años presentamos con el profesor Carlos Sirio un relato en un congreso argentino en el cual el tema eran los injertos ovári$\cos$. En ese momento nuestro entusiasmo fue grande y trabajamos en la forma experimental y clínico-experimental, y llegamos a la conclusión de que la supervivencia del ovario implantado aun con los requerimientos más intensos es escasa, y nunca llega a desarrollar una función ovárica perfectamente normal.

Los casos que más duraron con ciclos normales fueron de 8 meses y luego entraron en amenorrea. De modo que el ovario no respondía en la mayoría de los casos, y cuando lo hacía era por unos pocos meses.

Doctor Guerrero: Me pregunto si es necesario hablar de esto ahora que ya 
se conocen las hormonas, y no creo que se justifique aunque no tengo ninguna experiencia en esé campo.

Pregunta: ¿Cuánto puede fijar como límite preciso de tiempo entre una oligomenorrea y una amenorrea?

Doctor Guerrero: En México, p. e., por oligomenorrea se entiende la regia que dura pocos días; e hipomenorrea, la de poca cantidad. Podemos llamar regla retrasada poco, o mucho, para pensar en que cuando sea un mes, entonces ya es amenorrea. Pero en rigor el mecanismo es el mismo según se retrase 10 días o un mes, por las causas que se han expuesto; y las que duran más de un mes es que ya tienen otro tipo de causa. De manera que no encuentro diferencia para poner términos distintivos, es pura cosa de palabras.

Doctor Murray: Otro hecho de más importancia que el tiempo de amenorrea, es el de si la enferma tiene un ritmo de aparición de menstruaciones o seudo-menstruaciones. No sabemos aún lo que pueden ser, sin estudiar la enferma; en ese caso serán oligomenorreas así se trate de intervalos largos, dos meses o tres; es bastante frecuente en el ciclo monofásico ver ritmos de sesenta a noventa días. De modo que si nos atenemos a un período estricto de dos meses, ya entraría en amenorreas repetidas. En nuestro concepto es de catalogar a estas enfermas que tienen un ritmo menstrual o un ritmo de sangre, aunque sea una metrorragia, como oligomenorreicas. No se tráta pues de la duración de la amenorrea, sino de la existencia o no del ritmo.
Doctor López-Escobar: Yo estoy de acuerdo con el doctor Murray, y en esto hemos insistido durante largo tiempo, que lo importante es el ritmo, porque aun aquellas personas que insisten en que uno no debe definir una amenorrea sino una suspensión de noventa días, hablando corrientemente entre médicos se refieren, p.e., a una paciente "que tenía cuarenta días de amenorrea". De tal manera que indudablemente es más importante el ritmo que el tiempo.

Doctor Di Paola: Esta terminología que se refiere a los trastornos merece el ser reconsiderada. Hay un médico mexicano que me ha enviado un trabajo hace algún tiempo, donde él hace una nueva reestructuración de todos los términos de los sistemas menstruales; porque habría que empezar por revisar toda la terminología.

Quiero decir con este motivo que en este momento uno de los Comités designados por la Federación Internacional de Ginecología y Obstetricia, cuya reunión se organizó en Ginebra la semana pasada, encabezado por el profesor Louros, se ha encargado de estudiar una nueva nomenclatura para todos los términos que están en discusión; esta es una tarea que inició en primer lugar el profesor YAI del Japón, quien cuando llegó al Nơ 50 renunció, porque ya no podía más. Entonces la tomó el doctor Louros, que está ocupándose en el momento de esta cuestión. El doctor Louros quería hacer un diccionario de los términos ginecológicos en general, pero en vista de que para semejante obra serían necesarias por lo menos tres vidas consagradas a este propósito, 
se estableció que sólo se ocupara, como se ha venido haciendo hasta ahora, de los términos que provocan discusión, y ya se van a publicar las definiciones de los cincuenta primeros, que ya están hechas.

Con el problema ya de las seudo-amenorreas, nosotros, claro, como han dicho todos los compañeros de la mesa, cuando una enferma tiene la menstruación cada cuarenta y cinco o cada cincuenta días, es una oligomenorrea; porque la mayor parte de estas enfermas son de ciclo bifásico sin embarazo, y no tienen ningún problema, hasta que el médico no se lo produce. De modo que una cosa es la oligomenorrea con ciclo bifásico y con ovulación; y otra cosa es la oligomenorrea con ciclo monofásico, sin retraso menstrual, a la cual ya se ha referido el profesor Murray. Este distingo me parece importante, pues si una mujer siempre ha tenido un ritmo bifásico, no se puede considerar como una amenorrea sino como oligomenorrea. Ahora, si una mujer ha tenido siempre una regla normal y bruscamente hace un retraso, cómo no lo vamos a llamar amenorrea, si no es tragedia diaria, y así lo denominamos desde el punto de vista oficial y legal.

Doctor Guerrero: No estoy en ninguna forma en desacuerdo desde luego, pero ya que están hablando de ese Comité, del que yo formé parte en México, en la respuesta al doctor japonés insistimos en este punto de vista: con relación al término oligomenorrea, estoy oyendo la idea de nuestros compañeros argentinos, olygos en griego quiere decir poco. Pero ¿poco qué? ¿pocas menstruaciones en el año, o poca sangre durante las menstruaciones? Son dos enfoques diferentes. Yo creo que deberíamos nombrar un sub-comité que establezca una terminología para Latinoamérica, ya que todos hablamos el mismo idioma, porque todo aquelio que se decida para las definiciones puede no resultar siempre en español, ya que algunas palabras que son las mismas, digamos en inglés y en español, no significan lo mismo, y vamos a exponernos a que nos traigan una terminología que no esté en congruencia con el español.

Doctor Di Paola: Yo le quisiera decir al doctor Guerrero lo siguiente: $\mathrm{Si}$ él sostiene que olygos es poca menstruación, lo opuesto, polimenorrea, ¿qué es? Francamente me parece que nuestro punto de vista es el más lógico: oligomenorrea, pocas menstruaciones durante el año, y Polimenorrea, muchas menstruaciones durante el año.

Doctor Guerrero: Y nosotros 1lamábamos plurimenorrea a las muchas menstruaciones durante el año.

Al doctor Di Paola: Resultados en el tratamiento del Síndrome de Sheehan.

Doctor Di Paola: En primer lugar nosotros creemos que se llama enfermedad de Simmonds y no Síndrome de Sheehan. Esto fue un descubrimiento que hizo el doctor Simmonds, que es alemán, de Hamburgo, quien en una autopsia que practicó en una enferma, no me acuerdo exactamente en qué año, descubrió que dicha enferma tenía la hipófisis completamente atrofiada; entonces esta enfermedad de Simmonds, que ha sido estudiada por el inglés 
Sheehan, muy a fondo, pero no por docrinología. No vienen a los nuestros, eso se justifica que se le sustituya salvo raras excepciones. Se hace un trael nombre de su descubridor por el suyo. tamiento sustitutivo completo, dando Bueno, el tratamiento que se hace todas las hormonas que falten; es decir, para esta Enfermedad de Simmonds es se les da tiroides, cortisona, y hormoun tratamiento sustitutivo completo. nas sexuales. No puedo dar más opiNosotros no tenemos una gran expe- nión que ésta, pues no me gusta hablar riencia, ya que este tipo de enfermas en teoría, sino en lo que he visto $y$ generalmente van a los Servicios de En- conozco personalmente. 